\title{
La nueva regulación del trabajo a distancia no es la panacea
}

\author{
The new regulation of remote work isn't the "panacea" \\ Dra. Cristina AYALA DEL PINO \\ Universidad Rey Juan Carlos \\ Madrid \\ cristina.ayala@urjc.es \\ orcid: 0000-0002-2633-2973
}

\begin{abstract}
Resumen: La situación derivada de la declaración del estado de alarma provocado por la pandemia causada por el covid-19 supuso un gran cambio en el ámbito laboral; de repente el trabajo presencial se convirtió en teletrabajo. Esta situación inesperada propició la aprobación del Real Decreto-Ley 28/2020, de 22 de septiembre, de trabajo a distancia; regulación vigente que dota del calificativo digno al trabajo a distancia, haciendo hincapié, en aspectos tales como, su voluntariedad, riesgos laborales, el derecho a la desconexión y el papel fundamental que va a desempeñar la negociación colectiva en el trabajo a distancia.
\end{abstract}

Palabras claves: teletrabajo, trabajo a distancia, flexibilidad.

\begin{abstract}
The situation derived from the declaration of the state of alarm caused by covid-19 supposed a great change in the laboral ambit. Suddenly face to face work turned telework. This expected situation propitiated the approval of Royal Decree-Law 28/2020, of 22 of september, remote work; current regulation that endows of qualifying worthy to the remote work; emphasizing in aspects such as his willfulness, the occupational hazards, right to disconnect and the fundamental role is going to play the collective bargaining in the remote work.
\end{abstract}

Keywords: Telework, remote work, flexibility.

\section{Sumario:}

\section{Introducción.}


II. Concepto y regulación del trabajo a distancia y, su modalidad, el teletrabajo.

III. Requisitos necesarios para trabajar a distancia.

IV. Derechos de las personas trabajadoras a distancia.

\subsection{La formación.}

4.2. Tiempo de trabajo: horario flexible y registro de horario adecuado.

4.3. Prevención de riesgos en el trabajo a distancia.

4.4. Derecho a la desconexión digital en el trabajo a distancia.

4.5. Derechos colectivos de los trabajadores a distancia.

V. Control empresarial en el trabajo a distancia.

\section{Conclusiones.}

VII. Bibliografía.

Recibido: septiembre 2021. Aceptado: noviembre 2021. 


\section{INTRODUCCIÓN}

Ciertamente la intensa expansión del teletrabajo durante la pandemia derivada del Covid-19 ha impactado con mucha fuerza sobre la organización de numerosas empresas y trabajadores. Durante el confinamiento se incrementó el número de personas trabajando a distancia. El teletrabajo ha venido para quedarse, si bien no en las condiciones en las que se vino desarrollando en este periodo excepcional. Nadie cuestiona que el teletrabajo ha constituido una tabla de salvación frente a una emergencia de gran restricción de la movilidad, al declararse el Estado de alarma. Sin embargo, con vistas al futuro hay que pensar en un modelo que proporcione todo lo que de bueno tiene el teletrabajo. En este sentido, el modelo prevalente que se vislumbra es uno intermedio de semipresencialidad; esta fórmula puede ofrecer lo bueno que tiene el teletrabajo y lo positivo del trabajo presencial.

Cuando hayamos superado el escenario de la covid-19, en una situación de normalidad, el teletrabajo no cabe duda que puede aportar ventajas para las empresas y beneficios para los trabajadores; pero también resulta indiscutible que presenta riesgos y posibles efectos negativos para la productividad y para la garantía de los derechos de los trabajadores. Por ello, uno de los retos es conseguir un equilibrio entre las ventajas del teletrabajo y sus riesgos, que los hay.

De ahí que la aprobación el Real Decreto-Ley 28/2020, de 28 de septiembre, de trabajo a distancia cumpla un papel decisivo, ya que ha venido a cubrir una importantísima laguna de nuestra legislación laboral ${ }^{1}$, ya que no se había implantado el Acuerdo Europeo sobre la materia de 2002.

Esta norma ofrece una respuesta a los principales interrogantes que se planteaban en esta materia, pero al mismo tiempo hace hincapié en la flexibilidad que permita un amplio juego al pacto entre las partes y, sobre todo, a la negociación colectiva.

\footnotetext{
${ }^{1}$ Sobre la regulación anterior a este Real Decreto-Ley véase ALVÁREZ CUESTA, H., "Del recurso al teletrabajo como medida de emergencia al futuro del trabajo a distancia", en Lan Harremanak (Bizkaia), 43 (2020) 175 y ss.
} 
A pesar de que la norma cuenta con el aval de haberse alcanzado el equilibrio de intereses entre las partes a través de un nuevo acuerdo de concertación social. Todavía falta mucho por hacer, pues hay que llevar a la práctica lo previsto en la nueva norma. A tal fin, destacar que la negociación colectiva va a jugar un papel fundamental. De hecho, de ella va a depender que el nuevo modelo funcione con eficacia, ofreciendo protección a los trabajadores y proporcionando a los empresarios un modelo lo más flexible posible que les permita implantar sistemas de organización del trabajo conforme al uso generalizado de las nuevas tecnologías de la información de las comunicaciones, que han venido para quedarse y ayudarnos a desarrollar nuestro trabajo de la manera mejor posible.

\section{CONCEPTO Y REGULACIÓN DEL TRABAJO A DISTANCIA Y, SU MODALIDAD, EL TELETRABAJO}

A nivel internacional, la Organización Internacional del Trabajo aprobó el Convenio número 177, sobre trabajo a domicilio de 1996 y la Recomendación número 184, el cual entró en vigor el 22 de abril de 2020 y no ha sido ratificado por España todavía. Dicho convenio previó unos niveles de protección mínima para los trabajadores que prestaban su trabajo bajo esta modalidad haciendo hincapié en que esta prestación se caracteriza por la no coincidencia en espacio y tiempo con el empleador ${ }^{2}$.

En el ámbito comunitario, la Confederación Europea de Sindicatos, la Unión de Confederaciones de la Industria y de Empresarios de Europa, la Unión Europea del Artesanado y de la Pequeña y Mediana Empresa y el Centro Europeo de la Empresa Pública (CES, UNICE, UEAPME y CEEP, respectivamente) aprobaron el Acuerdo Marco Europeo sobre Teletrabajo, firmado el 16 de julio de 2002 en Bruselas y revisado en 2009 por los agentes sociales, el cual no comprometía directamente a los Estados, sino a las propias organizaciones empresariales de trabajadores firmantes a fin de dar más seguridad a los teletrabajadores y las teletrabajadoras por cuenta ajena en la Unión Europea. Este acuerdo entendió esta modalidad del trabajo a distancia como un medio para modernizar la organización del trabajo para las empresas y organizaciones de servicios públicos, y para dar una mayor autonomía en la realización de sus tareas a las personas trabajadoras. Dicho acuerdo define el teletrabajo como una forma de organización o de realización del trabajo utilizando las tecnologías de la información, en el marco de un contrato o de una relación laboral, en la

\footnotetext{
${ }^{2}$ Dicho Convenio entiende que esta modalidad se produce cuando se realiza la actividad laboral en el domicilio de la persona trabajadora o en otro local que ésta escoja, distinto de los locales de trabajo de la empresa, a cambio de una remuneración y con el fín de elaborar un producto o prestar un servicio conforme a las especificaciones de la misma.
} 
que un trabajo que también habría podido realizarse en los centros de la empresa, se ejecuta habitualmente fuera de $\operatorname{estos}^{3}$. En España no se llevó una incorporación efectiva al ordenamiento jurídico español, tan sólo se incorporó como anexo al Acuerdo Interconfederal para la Negociación Colectiva de 2005. No obstante, dicho acuerdo se ha presentado como un referente para los agentes sociales y la propia doctrina ${ }^{4}$.

En la regulación interna, hasta la aprobación de Real Decreto-Ley 28/2020, de Trabajo a distancia, la Ley 3/2012, de 6 de julio, de medidas urgentes para la reforma del mercado laboral, modificó la ordenación del tradicional trabajo a domicilio para dar acogida al trabajo a distancia basado en el uso intensivo de las nuevas tecnologías ${ }^{5}$. Conforme a esta modificación el precepto que regulaba su régimen jurídico de manera imprecisa e insuficiente era el artículo $13 \mathrm{ET}$, el cual no hacía referencia propiamente al teletrabajo, sino a una categoría más amplia del trabajo a distancia ${ }^{6}$. A partir de lo establecido en el Real DecretoLey se entiende por: "a) trabajo a distancia: forma de organización del trabajo o de realización de la actividad laboral conforme a la cual esta se presta en el domicilio de la persona trabajadora o en el lugar elegido por ésta, durante toda su jornada o parte de ella, con carácter regular; b) teletrabajo: aquel trabajo a distancia que se lleva a cabo mediante el uso exclusivo o prevalente de medios y sistemas informáticos, telemáticos y de telecomunicación; c) trabajo presencial: aquel trabajo que se presta en el centro de trabajo o en el lugar determinado por la empresa", conforme al artículo 2 de dicha norma ${ }^{7}$.

\footnotetext{
${ }^{3}$ En dicho Acuerdo se hace hincapié sobre las peculiaridades del teletrabajo y resalta aspectos tales como: el carácter voluntario del teletrabajo; la igualdad de derechos de las personas teletrabajadoras en relación a las que desarrollan su actividad en el establecimiento de la empresa; derecho a la formación y la carrera profesional, pleno ejercicio de sus derechos colectivos; la dotación de equipos; la seguridad y la salud; la gestión de la organización del trabajo por parte de la persona teletrabajadora, conforme a la legislación y convenios colectivos aplicables.

${ }^{4}$ Vid., SERRANO GARCÍA, J.M., "Tratamiento del teletrabajo en el Acuerdo Marco Europeo de 16 de julio de 2002", en Relaciones Laborales (Madrid), 2 (2002) 441-462.

${ }^{5} \mathrm{La}$ exposición de motivos de dicha Ley reconoció el teletrabajo como una particular forma de organización del trabajo que encaja en el modelo productivo y económico, al favorecer la flexibilidad de las empresas en la organización del trabajo, incrementar las oportunidades de empleo y optimizar la relación entre tiempo de trabajo y vida personal y familiar.

${ }^{6}$ El cual lo definía como aquel "en que la prestación de la actividad laboral se realice de manera preponderante en el domicilio del trabajador o en el lugar libremente elegido por este, de modo alternativo a su desarrollo presencial en el centro de trabajo de la empresa" (art. 13.1 ET).

${ }^{7}$ Las relaciones de trabajo a las que resulta de aplicación el Real Decreto Ley son aquellas en las que concurran las condiciones descritas en el artículo 1.1 ET, que se desarrollen a distancia con carácter regular. Entendiéndose por regular "el trabajo a distancia que se presente, en un periodo de referencia de tres meses, un mínimo del treinta por ciento de la jornada, o el porcentaje proporcional equivalente en función de la duración del contrato de trabajo" (art. 1 RD-Ley). Sobre la regulación del teletrabajo en las Administraciones Públicas véase BAQUERO
} 
Asimismo, hay que tener en cuenta el Real Decreto-Ley 6/2019 de 1 de marzo de medidas urgentes para garantía de la igualdad de trato y de oportunidades entre mujeres y hombres en el empleo y la ocupación, modificó el artículo 34.8 ET, anticipándose al contenido de la Directiva 2019/1158 del Consejo, de 20 de junio de 2019, relativa a la conciliación de la vida familiar y la vida profesional de los progenitores y los cuidadores y por la que se deroga la Directiva de la Unión Europea 2010/18 del Consejo, en la cual se establece un auténtico derecho a la conciliación de la vida laboral y familiar a través del uso de las formas flexibles de trabajo, incluidas las fórmulas de trabajo a distancia ${ }^{8}$.

Por su parte, la Ley Orgánica 3/2018, de 5 de diciembre, de Protección de Datos Personales y garantía de los derechos digitales, reconoce un conjunto de derechos relacionados con el uso de dispositivos en el ámbito laboral como son, entre otros, el derecho a la intimidad y uso de dispositivos digitales en el ámbito laboral y el derecho a la desconexión digital.

A pesar de la reciente regulación no cabe duda que la posibilidad de que el trabajador desempeñara su trabajo para el empresario fuera del centro de trabajo era una práctica reconocida por nuestro Derecho del Trabajo, si bien tal prestación debía estar sometida a algunas cautelas que evitaran que la falta de contacto físico entre trabajador y empleador pudiera derivar en abusos o arbitrariedades a la hora de prestar el trabajo, Sin embargo, el uso y difusión de la tecnología ha cambiado y seguirá haciéndolo la manera de trabajar, cambio que se ha visto acelerado por la situación de crisis en la que nos encontramos provocada por el Covid- $19^{9}$, constatando como real la posibilidad de trabajar sin coincidir en el mismo espacio físico -centro de trabajo-, ni con el resto de trabajadores ni con su empleador. Destacar que la negociación colectiva va a jugar un papel fundamental dentro del marco que establece el Real DecretoLey aprobado.

\footnotetext{
AGUILAR, J., "De la reciente regulación del teletrabajo en las Administraciones Públicas y de la necesidad de una precisa concreción vía negociación colectiva desde la perspectiva de género", en Revista Internacional y Comparada de Relaciones Laborales y Derecho del Empleo (México), ADAPT University Press, Vol. 8, nº 4 (2020) 106 y ss.

${ }^{8}$ Vid., GALA DURÁN, C., "La relación entre el teletrabajo y la conciliación de la vida laboral y familiar: el papel de la normativa y la negociación colectiva", en Anuario IET de Trabajo y Relaciones Laborales (Barcelona), 7 (2021) 171 y ss.

${ }^{9}$ A este respecto, el artículo 5 del Real Decreto-ley 8/2000, de 17 de marzo, de medidas urgentes extraordinarias para hacer frente al impacto económico y social del COVID-19, estableció el carácter preferente del trabajo a distancia frente a otras medias en relación con el empleo, debiendo la empresa adoptar las medidas oportunas si ello era técnica y razonablemente posible y si el esfuerzo de adaptación necesario resultaba proporcionado, tratándose de una norma excepcional y de vigencia limitada.
} 


\section{REQUISITOS NECESARIOS PARA TRABAJAR A DISTANCIA}

Respecto a la prestación de trabajo a distancia y, en su modalidad, teletrabajo conviene analizar las condiciones exigidas para su aplicación:

a) Forma escrita. Es necesario que el acuerdo de trabajo a distancia se realice por escrito. Dicho acuerdo puede estar incorporado al contrato de trabajo inicial o realizarse durante el transcurso del mismo. Ahora bien, debe formalizarse antes de que se inicie el trabajo a distancia (art. 6.1. RD-Ley). Este artículo no prevé qué sucede en el supuesto de la falta de forma, por lo que cabe entender en tal caso que la prestación de servicios se prestará en el centro de trabajo al que esté adscrito el trabajador.

Asimismo, el precepto expuesto recoge expresamente la obligación, por parte de la empresa, de entregar a la representación legal de las personas trabajadoras una copia de todos los acuerdos de trabajo a distancia que se realicen, como garantía en orden al control del cumplimiento de la normativa laboral.

b) Voluntario. La voluntariedad exigida en el trabajo a distancia implica la necesidad de requerir la firma del acuerdo de ambas partes para el desarrollo de la prestación de servicios en un lugar diferente ${ }^{10}$. Dicho acuerdo puede formar parte del contrato inicial o realizarse en un momento posterior, es decir, durante su transcurso.

El contenido de este precepto jurídico está en consonancia con lo previsto tanto en el artículo 3 del Acuerdo Marco Europeo como el artículo 6 del Convenio 177 OIT abogan por imponer las notas de libertad y voluntariedad para las partes, sin que quepa la posibilidad de sufrir el trabajador alguna consecuencia desfavorable por negarse a aceptar su realización ${ }^{11}$.

Es decir, la negativa de la persona trabajadora a trabajar a distancia, el ejercicio de la reversibilidad al trabajo presencial y las dificultades para el desarrollo adecuado de la actividad laboral a distancia, no han de calificarse como causas

${ }^{10}$ El empleador tiene diez días para entregarlo a los representantes de los trabajadores y después tendrá que remitirlo a la oficina de empleo.

${ }^{11}$ Literalmente el art. 3 del Acuerdo Europeo reconoce cómo "el teletrabajo es voluntario para el trabajador y el empresario afectados. El teletrabajo puede formar parte de la descripción inicial del puesto de trabajo o puede incorporarse de forma voluntaria más tarde... Si el teletrabajo no forma parte de la descripción inicial del puesto, y si el empresario hace una oferta de teletrabajo, el trabajador puede aceptarla o rechazarla. Si es el trabajador quien expresa su deseo de teletrabajar el empresario puede aceptarla o rechazarla. El paso al teletrabajo en tanto que tal, puesto que modifica únicamente la manera en que se efectúa el trabajo, no afecta al estatus laboral del trabajador. La negativa de un empleado a teletrabajar no es, en sí, un motivo de rescisión de la relación laboral ni de motivación de las condiciones de trabajo de este trabajador". 
justificativas de la extinción de la relación laboral ni de la modificación sustancial de las condiciones de trabajo ${ }^{12}$.

El artículo 5.3 del Real Decreto-Ley reconoce que la decisión de trabajar a distancia desde la modalidad de trabajo presencial es reversible para la empresa y la persona trabajadora. El ejercicio de dicha reversibilidad se ejercerá conforme a la negociación colectiva o, en su defecto, en los términos fijados en el acuerdo de trabajo (art. 7 RD-Ley).

De todo lo expuesto, se deduce que el trabajo en remoto será voluntario para el empleado y la empresa. También es reversible, y este derecho se tiene que ejercer en función de lo que fije la negociación colectiva o el acuerdo firmado entre empresario y trabajador. Se prevé asimismo que será voluntaria -para ambas partesla modificación del porcentaje de presencialidad, que tendrá que llevarse a cabo de acuerdo con lo establecido en el convenio colectivo o el acuerdo de empresa.

Por último, aunque los detalles se establecerán conforme con la negociación colectiva, cabe destacar que el acuerdo de trabajo a distancia debe recoger el siguiente contenido mínimo con carácter obligatorio: entre los doce puntos que destaca el texto están el inventario de los medios, equipos y herramientas necesarias para desarrollar la actividad en remoto, incluidos los consumibles y los elementos muebles; la enumeración de los gastos en los que puede incurrir el trabajador y la modalidad para cuantificarlos y compensarlos; el horario de trabajo o reglas de disponibilidad; porcentaje y distribución; el centro del trabajo; el lugar de trabajo elegido para teletrabajar; duración de plazos de preaviso para ejercer la reversibilidad; los medios de control empresarial; el procedimiento a seguir en caso de producirse dificultades técnicas; las instrucciones dictadas por la empresa para ejercer la actividad; la duración del acuerdo. También entraría en este acuerdo las instrucciones de la empresa con la participación de los representantes de los trabajadores, en materia de protección de datos y sobre seguridad de la información.

\section{DERECHOS DE LAS PERSONAS TRABAJADORAS A DISTANCIA}

\subsection{La formación}

La formación de los trabajadores a distancia constituye un derecho esencial en dos sentidos: por una parte, aquella permite a los trabajadores seguir

${ }^{12}$ Al respecto véase la STS de 11 de abril de 2005, Rec. núm. 143/2004, en virtud de la cual se declara que no cabe modificar esta condición laboral a través del art. 41 ET, en tanto trasformaría la naturaleza del vínculo. 
avanzando en su carrera profesional, al tener las mismas oportunidades que el resto de sus compañeros que presentan sus servicios en el centro de trabajo en modalidad presencial y ligada a la promoción profesional; y por otra, hace referencia a la que sea necesaria para el correcto desempeño de la relación laboral a distancia, con el fin de cumplir con las exigencias del trabajo a desempeñar.

A este respecto, el artículo 9.1. Real Decreto-Ley exige que las empresas establezcan "las medidas necesarias para garantizar la participación efectiva en las acciones formativas de las personas que trabajan a distancia, en términos equivalentes a las de las personas que prestan servicios en el centro de trabajo de la empresa, (...)". El contenido de este precepto pretende evitar que los trabajadores a distancia al estar en una situación de aislamiento puedan quedarse obsoletos profesionalmente, en la medida de lo posible hay que evitar que sufran pérdida de habilidades y competencias como consecuencia de la distancia con respecto a los centros de trabajo de las empresas. Asimismo, las personas que trabajan a distancia tienen derecho, "en los mismos términos que las que prestan servicios de forma presencial, a la promoción profesional, debiendo la empresa informar a aquellas, de manera expresa y por escrito, de las posibilidades de ascenso que se produzcan, ya se trate de puestos de desarrollo presencial o a distancia" (art. 10 RD-Ley) ${ }^{13}$. El contenido de este artículo, hace hincapié en que todos los trabajadores presenciales o a distancia tienen derecho a ser informados de las posibilidades de ascenso, garantizando así que pueden concurrir en igualdad de condiciones ${ }^{14}$.

Por otra parte, la empresa debe "garantizar a las personas que trabajan a distancia la formación necesaria para el adecuado desarrollo de su actividad tanto al momento de formalizar el acuerdo de trabajo a distancia como cuando se produzcan cambios en los medios o tecnologías utilizadas" (art. 9.2 RDLey $)^{15}$. El contenido de este precepto es relevante si se desea ver al trabajo a distancia como modalidad de trabajo digno y decente.

${ }^{13}$ El artículo vigente, una vez más, recoge lo previsto en el Acuerdo Marco Europeo, que precisó más el derecho a la formación, ante la posibilidad de caer en el olvido para futuros ascensos al no encontrarse físicamente en el centro del trabajo, reconociendo que "los trabajadores tienen el mismo acceso a la formación y a las posibilidades de carrera profesional que trabajadores similares que realizan su tarea en los locales del empresario, y están sujetos a las mismas políticas de evaluación que los demás trabajadores”.

14 Vid., DE CASTRO MEJUTO, L.F., "La formación y promoción profesional en el teletrabajo", en Trabajo a distancia y teletrabajo: estudios sobre el régimen jurídico en el derecho español y comparado, Coords. Alicia Villalba Sánchez y Lourdes Mella Méndez, Aranzadi, Pamplona 2015, p. 95.

${ }^{15}$ Este apartado prevé lo que ya precisaba el Acuerdo Marco Europeo al reconocer que los trabajadores a distancia tienen derecho a recibir una formación adecuada "para utilizar el 
Por último, cabe señalar que ni la norma vigente ni el Acuerdo Marco Europeo, hacen referencia al contenido, la modalidad o la duración de la formación requerida, lo cual resulta lógico, ya que dependerá de cada puesto de trabajo y sector, siendo su desarrollo competente, el convenio colectivo aplicable en rasgos generales.

\subsection{Tiempo de trabajo: horario flexible y registro de horario adecuado}

La norma establece que la empresa tiene que encargarse de la dotación y mantenimiento de los medios y equipos que necesita el trabajador a distancia para trabajar, en los términos establecidos en la negociación colectiva o en el acuerdo de teletrabajo (art. 11 RD-Ley). Asimismo, el texto señala que el desarrollo de la actividad tiene que ser sufragado o compensado por la empresa, ya que el trabajador no puede asumir los gastos relacionados con los equipos y el resto de medios que le hacen falta para realizar su actividad (gastos fijos de electricidad, red, etc.,). Los convenios o acuerdos colectivos podrán establecer el mecanismo para determinar y compensar estos gastos (art. 12 RD-Ley).

\subsection{Tiempo de trabajo: horario flexible y registro del horario adecuado}

El hecho de desarrollar la prestación laboral a través del trabajo a distancia no afecta a la duración de la jornada. Ahora bien, sí puede afectar a ciertos aspectos tales como el horario, es decir, al momento exacto en que la prestación es debida ${ }^{16}$, máxime cuando en la mayoría de las ocasiones el teletrabajo hace referencia al trabajo flexible a distancia. En este sentido, el artículo 13 Real Decreto-Ley reconoce que conforme al "acuerdo de trabajo a distancia y la negociación colectiva, respetando los tiempos de disponibilidad obligatoria y la normativa sobre tiempo de trabajo y descanso, la persona que desarrolla trabajo a distancia podrá flexibilizar el horario de prestación de servicios establecido".

Asimismo, el trabajador tiene derecho al registro del horario adecuado. El propio Real Decreto-Ley se remite, en este sentido, al artículo 34.9 ET cuando

equipo técnico a su disposición y sobre las características de esta forma de organización del trabajo". Es más, su contenido va más allá y hace referencia a la necesidad de dar oportuna formación tanto al supervisor de los teletrabajadores como a sus compañeros de trabajo para adecuarse a esta forma de trabajo y saber gestionarla.

${ }^{16}$ En este sentido, véase SUÁREZ QUIÑONES, J.C., "La organización del tiempo de trabajo y descanso y la conciliación en el teletrabajo", en Trabajo a distancia y teletrabajo, Aranzadi, Pamplona 2016, p. 133. 
se refiere al sistema de registro horario ${ }^{17}$, reconociendo que conforme a lo previsto en la negociación colectiva, "deberá reflejar fielmente el tiempo que la persona trabajadora que realiza trabajo a distancia dedica a la actividad laboral, sin perjuicio de la flexibilidad horaria, y deberá incluir, entre otros, el momento de inicio y finalización de la jornada" (art. 14 RD-Ley).

No cabe duda que la recogida de los datos de los trabajadores a distancia, tendrá que realizarse de manera telemática y remota, así como de forma objetiva y no modificable, mediante los instrumentos informáticos que se utilizan para el desarrollo de la prestación laboral ${ }^{18}$.

Al respecto, cabe destacar que la utilización de un sistema de registro de horario va destinado a evitar la fijación del trabajo por objetivos, lo que se denomina "destajo tecnológico"19 o la siempre disponibilidad, haciendo referencia al "presentismo digital". De hecho la flexibilidad del teletrabajo puede ser diseñada, siempre y cuando el tiempo trabajado quede registrado ${ }^{20}$, lo que se puede hacer de manera sencilla a través de medios técnicos.

En suma, el decreto establece que se pueden fijar unos tiempos de disponibilidad obligatoria. Esto es, se reconoce la flexibilidad horaria -según lo establecido en el acuerdo de teletrabajo y la negociación colectiva, y siempre y cuando se respete la normativa sobre tiempo de trabajo y descanso-, pero a la vez

${ }^{17}$ En virtud de este artículo y apartado, la empresa ha de garantizar "el registro diario de jornada, que deberá incluir horario concreto de inicio y finalización de la jornada de trabajo de cada persona trabajadora, sin perjuicio de la flexibilidad horaria (...) Mediante negociación colectiva o acuerdo de empresa o, en su defecto, decisión del empresario previa consulta con los representantes legales de los trabajadores en la empresa, se organizará y documentará este registro de jornada. La empresa conservará los registros a que se refiere este precepto durante cuatro años y permanecerán a disposición de las personas trabajadoras, de sus representantes legales y de la Inspección de Trabajo y Seguridad Social".

${ }^{18}$ Vid., TASCÓN LÓPEZ, R., "El derecho de desconexión del trabajador (potencialidades en el ordenamiento español)", en Trabajo y Derecho (Madrid), 41 (2018) 45-63.

${ }^{19}$ En este sentido, véase SIERRA BENÍTEZ, E.M., "la retribución, suspensión y extinción en el teletrabajo", en Trabajo a distancia y teletrabajo, Cizur Menor, Aranzadi, Pamplona 2016, p. 211.

${ }^{20}$ Cfr., la STSJ Castilla y León/Valladolid de 3 de febrero de 2016, Rec. núm. 2229/2015, en virtud de la cual se entiende que "solamente si la empresa ha establecido pautas claras sobre tiempo de trabajo respetuosas con la regulación legal y convencional sobre jornada y descansos y si además establece, de acuerdo con el trabajador, instrumentos de declaración y control del tiempo de trabajo a distancia o en el domicilio, sería posible admitir que una conducta del trabajador en el interior de su domicilio en vulneración de dichas pautas y omitiendo los instrumentos de control empresarial pudiera dar lugar a exceptuar el pago de las correspondientes horas y su cómputo como tiempo de trabajo. Pero en ausencia de esas pautas y criterios y de unos mínimos instrumentos de control no puede admitirse tal exceptuación, que sería equivalente a crear un espacio de total impunidad y alegalidad en el trabajo a distancia y en el domicilio". 
empresario y trabajador pueden negociar si en determinadas franjas horarias el empleado tiene que garantizar su disponibilidad. También se mantiene el sistema de registro horario, que deberá incluir el momento de inicio y finalización de la jornada y de los tramos de actividad.

\subsection{Prevención de riesgos en el trabajo a distancia}

La protección de la salud y seguridad de los trabajadores a distancia viene garantizada en el artículo 15 Real Decreto-Ley en virtud del cual se reconoce la aplicación de la Ley 31/1995, de 8 de noviembre, de Prevención de Riesgos Laborales y sus normas de desarrollo ${ }^{21}$. De hecho este artículo reproduce prácticamente el contenido del artículo 13.4 ET que tradicionalmente ha sido calificado como demasiado ambicioso y poco efectivo ${ }^{22}$.

Este artículo trata de asegurar que ningún trabajador sufra daño alguno a la hora de prestar su prestación laboral ${ }^{23}$. Ahora bien, este deseo choca por una parte con el lugar de la prestación, éste se encuentra fuera del centro del trabajo y, por la otra, el teletrabajo se realizará en el domicilio de la persona trabajadora, cuya inviolabilidad incrementa la desprotección que dicho artículo pretende garantizar.

Por ello, es necesario que la persona teletrabajadora colabore o, incluso, pacta qué lugares se consideran más seguros para el teletrabajador a la hora de desarrollar la prestación de servicios ${ }^{24}$.

El nuevo Real Decreto-Ley va más allá y prevé la evaluación de riesgos y la planificación de la actividad preventiva del trabajo a distancia. De hecho, hay que tener en cuenta los riesgos característicos de esta modalidad de trabajo, sobre todo, los factores psicosociales, ergonómicos y organizativos que influyen en la misma, Así como, la distribución de la jornada, los tiempos de disponibilidad y la garantía de los descansos y desconexiones durante la jornada.

${ }^{21}$ Este artículo prácticamente reproduce el contenido del artículo 13.4 ET.

${ }^{22}$ En este sentido, véase TASCÓN LÓPEZ, R., "El teletrabajo como forma de presente y futuro de prestación laboral de servicios. Experiencias en la negociación colectiva", en El futuro del Trabajo: cien años de la OIT: comunicaciones, Ministerio de Trabajo, Migraciones y Seguridad Social, Subdirección General de información Administrativa y Publicaciones, Madrid 2019, pp. 1615 y ss

${ }^{23}$ Vid., SELMA PENALVA, A., "El accidente de trabajo en el teletrabajo. Situación actual y nuevas perspectivas", en Temas Laborales. Revista Andaluza de Trabajo y Bienestar social (Sevilla), 134 (2016) 129.

${ }^{24}$ En este sentido, véase MELLA MÉNDEZ, L., "La seguridad y salud en el trabajo", en Trabajo a distancia y teletrabajo: estudios sobre su régimen jurídico en el Derecho español y comparado, Cizur Menor, Aranzadi, Pamplona 2015, p. 171. 
De hecho, es una realidad que en esta modalidad de trabajo concurren riesgos específicos, tales como los ergonómicos (silla, mesa, etc.,) y ambientales (iluminación, temperatura, etc.,) y de los derivados del uso intensivo de pantallas de visualización ${ }^{25}$, pero también y no menos importantes los riesgos psicosociales coligados al trabajado aislado y ligado a la utilización excesiva e intensiva de la tecnología. Pero, además, hay que prestar especial atención a otros riesgos tales como la tecnoadicción (necesidad de estar permanentemente conectado), al tecnoestrés que provoca fatiga física y mental, dolores de cabeza, ansiedad; adición al trabajo o la depresión o ansiedad causadas por la soledad. De hecho, la hiperconectividad constante del trabajador incrementa los riesgos relativos a la fatiga informática que puede tener consecuencias para el trabajador tanto física como mentalmente. También hay que tener en cuenta trastornos como la nomofobia, entendida como miedo irracional a quedarse sin teléfono móvil y que puede generar en la persona una sensación de incomunicación cuando este no puede disponer de él. Los síntomas físicos que produce este trastorno son taquicardias, sudoración e incluso dolor de cabeza y estómago; la fomofobia, entendida como dependencia del móvil u otro dispositivo para trabajar, si bien, hay que hacer hincapié en que es una adicción relacionada con las redes sociales. En este sentido, juegan un papel esencial las disciplinas de la prevención de riesgos laborales que estudian la psicosocicología ${ }^{26}$ y la ergonomía del trabajo ${ }^{27}$.

Asimismo, el teletrabajo puede conllevar a que aparezcan problemas de salud física derivados de la vida sedentaria y la falta de actividad física, ya que el trabajo se realiza con ordenadores, siendo un hecho que puede incrementar el riesgo de accidentes cardiovasculares, obesidad, derrames cerebrales o diabetes; problemas ergonómicos u oftalmológicos derivados de estar siempre mirando a la pantalla con atención y posturas inadecuadas al estar sentados o con sillas no ergonómicas o la sobrecarga de información continua para procesar ${ }^{28}$.

De ahí que sea necesaria la evaluación de riesgos únicamente de la zona habilitada para la prestación de servicios, no extendiéndose al resto de zonas

${ }^{25}$ Cfrs., RD 488/1997, de 14 de abril, sobre disposiciones mínimas de seguridad y salud relativas al trabajo con equipos que incluyen pantallas de visualización y su guía técnica de desarrollo.

${ }^{26}$ A este respecto la Nota Técnica Preventiva 1122 del Instituto Nacional de Seguridad y Salud en el Trabajo incorpora un listado no exhaustivo de factores de riesgos psicosocial por el uso inadecuado y no planificado de las TIC's relacionados con el tiempo de trabajo.

${ }^{27}$ A este respecto, téngase en cuenta el Real Decreto 488/1997, de 14 de abril, sobre disposiciones mínimas de seguridad y salud relativas al trabajo con equipos que incluyen pantallas de visualización.

${ }^{28}$ Vid., FERNÁNDEZ PROL, F., "La "conectividad" del trabajador: análisis desde una óptica preventiva”, en Temas Laborales. Revista Andaluza de Trabajo y Bienestar (Sevilla), 156 (2021), 149 y ss. 
de la vivienda o del lugar elegido para el desarrollo del trabajo a distancia. Por ello, la empresa debe "obtener toda la información acerca de los riesgos a los que está expuesta la persona que trabaja a distancia (...) y prever las medidas de protección que resulten más adecuadas en cada caso" (art. 16.2 RD-Ley). Dicha información puede ser obtenida por parte de quien tenga competencias en materia preventiva (art. 7 RD-Ley), siempre y cuando se otorgue el permiso de la persona trabajadora y, en caso de que no se concediese, la información puede aportarla la propia persona trabajadora conforme a las instrucciones del servicio de prevención (art. 16.2 in fine RD-Ley). Es más, el trabajador podría acudir a su médico de cabecera, conocedor de sus patologías, para solicitar un reconocimiento para valorar si su perfil es idóneo para solicitar o adherirse a los programas de teletrabajo de su empresa, haciendo hincapié en las pautas de conductas y previsibles consecuencias para su salud ${ }^{29}$.

Por último, no cabe duda que habría que adaptar las condiciones de trabajo cuando quienes vayan a prestar sus servicios resulten muy sensibles a alguno de los riesgos mencionados, así como se debe garantizar no sólo la formación general para desarrollar el teletrabajo sino también en materia de prevención en riesgos laborales conforme a la evaluación realizada por la empresa.

\subsection{Derecho a la desconexión digital en el trabajo a distancia}

Conforme al artículo 18. Real Decreto-Ley "las personas que trabajan a distancia, particularmente en teletrabajo, tienen derecho a la desconexión digital fuera de su horario de trabajo" (art. 18.1) conforme al artículo 88 LO 3/2018, de 5 de diciembre, de Protección de Datos Personales y Garantía de los Derechos Digitales ${ }^{30}$.

A este respeto, dicho artículo reproduce el contenido del artículo $88 \mathrm{LO}$ 3/2018. Así pues, el deber del empresario de garantizar dicha desconexión

${ }^{29}$ En este sentido, véase MELLA MÉNDEZ, L., "La seguridad y salud...”, o. c., p. 171.

${ }^{30}$ Este derecho de índole digital entró en vigor en España el 7 de diciembre de 2018, tomando forma en la Ley Orgánica 3/2018, de 5 de diciembre, de Protección de Datos Personales y Garantía de los Derechos Digitales. En este sentido, de conformidad con lo previsto en el artículo 88 LO 3/2018 "los trabajadores y los empleados públicos tendrán derecho a la desconexión digital a fin de garantizar, fuera del tiempo de trabajo legal o convencionalmente establecido, el respeto de su tiempo de descanso, permisos y vacaciones, así como de su intimidad personal y familiar". Esta norma introdujo en el Estatuto de los Trabajadores un artículo 20 bis que recalca que los empleados "tienen derecho a la intimidad en el uso de los dispositivos digitales puestos a su disposición por el empleador, a la desconexión digital y a la intimidad frente al uso de dispositivos de vigilancia y geolocalización en los términos establecidos en la legislación vigente en materia de protección de datos y garantía de los derechos digitales". 
"conlleva una limitación del uso de los medios tecnológicos de comunicación empresarial y de trabajo durante los periodos de descanso, así como el respeto a la duración máxima de la jornada y a cualesquiera límites y precauciones en materia de jornada que dispongan la normativa legal o convencional" (art. 18.2 segundo párrafo RD-Ley). El contenido de este artículo se justifica porque como sabemos el uso de la tecnología nos hace perder la noción del tiempo y lugar, difuminándose las fronteras entre el tiempo de trabajo efectivo del tiempo de ocio y descanso, llegando incluso a llevarnos a estar en conexión permanente sin darnos cuenta o provocando que el empleado nunca desconecte del trabajo. Y ello es así porque la utilización de las Tecnologías de la Información y Comunicación han empujado a una disponibilidad horaria y locativa constante de los trabajadores, incluso, fuera de su horario laboral. Por ello, sería conveniente que en materia de prevención de riesgos laborales se blindara el derecho al descanso en forma de desconexión. Es más, esta conectividad continua lleva al trabajador a no desconectar nunca de su trabajo, con las consecuencias negativas que pueden sufrir los trabajadores desde su seguridad y salud, así como, los riesgos psicosociales han de ser valorados por las empresas dando cumplimiento a la acción preventiva prevista en la Ley 31/1995, de 8 de noviembre, de prevención de riesgos laborales ${ }^{31}$.

De ahí que la norma obligue a las empresas, previa audiencia de la representación legal de las personas trabajadoras, a elaborar una "política interna dirigida a personas trabajadoras, incluidos los que ocupen puestos directivos, en la que definirán las modalidades de ejercicio del derecho a la desconexión $\mathrm{y}$ las acciones de formación y de sensibilización del personal sobre un uso razonable de las herramientas tecnológicas que evite el riesgo de fatiga informática". Y en particular, preservarán "el derecho a la desconexión digital en los supuestos de realización total o parcial del trabajo a distancia, así como en el domicilio de la persona empleada vinculado al uso con fines laborales de herramientas tecnológicas" (art. 18.2 RD-Ley). Esta mención se justifica por la interconexión entre teletrabajo y trabajo flexible, de manera que la desconexión digital actuaría como frontera para delimitar este trabajo del descanso semanal, interjornadas o las propias vacaciones. Asimismo, dicho artículo encomienda a la negociación colectiva establecer "los medios y medidas adecuadas para garantizar el ejercicio efectivo del derecho a la desconexión en el trabajo a distancia y la organización adecuada de la jornada de forma que sea compatible con la garantía de tiempos de descanso".

Dicho artículo constata el papel fundamental que desempeña la negociación colectiva, máxime cuando el derecho a la desconexión digital supone reconocer

${ }^{31}$ En extenso, véase TRUJILLO PONS, F., "La "desconexión digital" a lomos de la seguridad y salud en el trabajo”, en Lan Herremanak (Bizkaia), 45 (2021) 1-18. 
el derecho al descanso en un mundo en el que vivimos hiperconectados, que si bien atiende a la naturaleza y objeto de la relación laboral, también ha de garantizar el derecho a la conciliación de la actividad laboral y la vida personal. Por ello, habrá que estar atentos a comprobar cómo los convenios colectivos regulan este derecho ya que con anterioridad al Real Decreto-Ley, realizaban un vago reconocimiento del derecho de manera indeterminada ${ }^{32}$, sin concretar el deber de abstención del empresario y sin medios e instrumentos para hacerlo efectivo $^{33}$, dejando a voluntad del trabajador no contestar correos o mensajes. Quizás los próximos convenios colectivos deberían establecer la obligación de "no enviar correos ni mensajes" en lugar de no contestar a voluntad del trabajador, establecer mecanismos que impidan recibir comunicación del trabajo; o la desconexión total y automática, o el bloqueo a herramientas digitales, fijar períodos de descanso digital o fijar una hora para la desconexión, etcétera.

En definitiva, si las empresas pusieran en la práctica el ejercicio de este derecho, la carga mental y fatiga informática del trabajador no se vería afectada por unos niveles que pudieran repercutir en la salud de los trabajadores. Sin embargo, la realidad nos constata que las empresas incumplen de manera sistemática este derecho. Por ello, sería conveniente que se desarrollara reglamentariamente la actual normativa, de hecho, es necesario una regulación más amplia del derecho de desconexión digital en el trabajo, máxime cuando este derecho ha venido para quedarse y ocupar una posición muy relevante en el ámbito de las relaciones laborales.

\subsection{Derechos colectivos de los trabajadores a distancia}

Conforme al artículo 19 Real Decreto-Ley los trabajadores a distancia tienen "derecho a ejercitar sus derechos de naturaleza colectiva con el mismo contenido y alcance que el resto de las personas trabajadoras del centro al que están adscritas". El contenido de este artículo trata de neutralizar las diferencias entre trabajo presencial y trabajo a distancia ${ }^{34}$.

A este respecto, la negociación colectiva "podrá establecer las condiciones para garantizar el ejercicio de los derechos colectivos de las personas trabajadoras

32 Vid., IGARTÚA MIRÓ, T., "El derecho a la desconexión en la Ley orgánica 3/1988, de 5 de diciembre, de protección de datos personales y garantía de los derechos digitales", en Revista de Trabajo y de la Seguridad Social, CEF (Madrid), 452 (2019) 80.

${ }^{33}$ En este sentido, véase GARCÍA JIMÉNEZ, M., "Revolución industrial 4.0, sociedad cognitiva y relaciones laborales: retos para la negociación colectiva en clave de bienestar de los trabajadores", en Revista de Trabajo y de la Seguridad Social, CEF (Madrid), 432 (2019) 172.

${ }^{34}$ En este sentido, véase PASTOR MARTÍNEZ, A., "Trabajo a distancia y derechos colectivos en el Real Decreto-Ley 28/2020 de trabajo a distancia”, en Anuario IET de Trabajo y Relaciones Laborales (Barcelona), 7 (2021) 110 y ss. 
a distancia". Esta representación colectiva es necesaria dado el individualismo que conlleva la prestación laboral a distancia al no estar en el centro de trabajo físico y la dificultad que hay en establecer relaciones colectivas máxime cuando estamos inmersos en un mundo digitalizado ${ }^{35}$. Es más, dicha representación debería jugar un papel fundamental para garantizar la solidaridad necesaria para construir la negociación y abogar por la promoción de intereses comunes, haciendo visibles a dichos trabajadores a pesar de trabajar a distancia, fomentándola.

Para facilitar esta negociación, la empresa asume el deber de suministrar a la representación legal de los trabajadores a distancia los elementos que sean "precisos" y necesarios para el desarrollo de su actividad representativa, entre otros, el acceso a las comunicaciones electrónicas de uso en la empresa y la existencia del tablón virtual. Asimismo, debe asegurarse que no haya obstáculos para la comunicación entre los trabajadores a distancia y sus representantes legales ${ }^{36}$. Asimismo, la organización empresarial tiene que garantizar que estos trabajadores pueden participar en las actividades organizadas o convocadas por su representación legal o por otros trabajadores en defensa de sus intereses laborales, sobre todo, hay que asegurar su participación efectiva presencial para el ejercicio del derecho a voto en las elecciones a representantes legales ${ }^{37}$.

Puede pensarse que al no existir un centro de trabajo dificulta la adscripción de los trabajadores a un determinado centro para elegir a sus representantes y poder llevar a la práctica la negociación colectiva estatutaria. El empresario, por regla general, configura a su arbitrio las circunstancias que permiten calificar una unidad productiva como centro de trabajo, siendo su libertad muy amplia ${ }^{38}$. En este sentido, se podría considera aquel en el que el trabajador realiza parte de su prestación en un único centro, en cuyo caso el trabajador sería adscrito a aquel ${ }^{39}$. Ahora bien, si no existiera centro de trabajo y, los trabajadores no

${ }^{35}$ Vid., ROJO TORRECILLA, E., "Cambios en el mundo del trabajo. El ejercicio de los derechos colectivos en el entorno empresarial digital y la adaptación sindical", en El nuevo y cambiante mundo del trabajo. Una mirada abierta y crítica a las nuevas realidades laborales",

htpp://www.eduardorojotorrecilla.es/2019/06/cambios-en-el-mundo-del.trabajo-el.html, 3 de junio de 2021.

${ }^{36}$ En este sentido, véase PASTOR MARTÍNEZ, A., "Trabajo a distancia...”, o. c., pp.113 y ss.

${ }^{37}$ Ibídem, p. 119.

${ }^{38}$ En este sentido, ÁlVAREZ DEL CUVILLO, A., "El centro de trabajo como unidad electoral: un concepto jurídico indeterminado en un contexto de cambio organizativo", en Nueva Revista Española de Derecho del Trabajo (Pamplona), 188 (2016) 141.

${ }^{39}$ Vid., PASTOR MARTÍNEZ, A., "Una aproximación a la problemática de la representación colectiva de los trabajadores de las plataformas "colaborativas" y en entornos virtuales", en IUS Labor (Barcelona), 2 (2018) 227, https://doi.org/10.31009 IUSlabor.2018.io2.06 [consulta 25-5-2021]. 
realizaran sus servicios en uno, tendrían que estar adscritos a un centro, en tal caso, podrían ser agrupados todos a uno o repartidos en varios. Es más, sería posible delimitar a los trabajadores adscritos a un centro por su acceso a la aplicación y su operatividad en un espacio físico determinado ${ }^{40}$, así se evitaría cercenar el derecho de representación y negociación.

Asimismo, puede pensarse que al no existir un centro de trabajo físico resulta difícil llevar a cabo tareas tales como la publicación del censo, de la lista de candidatos o la propia celebración de elecciones en un espacio concreto ${ }^{41}$, si el trabajador a distancia no está en el centro del trabajo donde se prestan los servicios.

Ciertamente, pueden existir estos obstáculos para garantizar estos derechos al prestarse el trabajo a distancia, pero el empresario ha de garantizar los medios que faciliten y favorezcan el desarrollo de los mismos, para ello, deberá utilizar los medios digitales y la tecnología existente que permitan su ejercicio conforme a la normativa vigente comentada anteriormente.

\section{CONTROL EMPRESARIAL EN EL TRABAJO A DISTANCIA}

Hoy en día nadie cuestiona las variadas posibilidades de control del empresario del trabajo a distancia realizado por los trabajadores dadas las infinitas posibilidades que bridan la tecnología actual a través de la utilización de seguidores URL, a través de los cuales se puede rastrear los movimientos de los trabajadores en la red, tecnología ASP que permiten conocer la ubicación de los trabajadores por teléfono móvil, sniffers o identificadores de radiofrecuencia RFID $^{42}$.

Al respecto, el artículo 22 Real Decreto-Ley reconoce que la empresa puede adoptar "las medidas que estime más oportunas de vigilancia y control para verificar el cumplimiento por la persona trabajadora de sus obligaciones y deberes laborales, incluida la utilización de medios telemáticos, guardando en su adopción y aplicación la consideración debida a su dignidad y teniendo en cuenta, en su caso, la capacidad real de los trabajadores con discapacidad"43.

\footnotetext{
${ }^{40}$ Vid., GARRIDO PÉREZ, E., "La representación de los trabajadores al servicio de las plataformas colaborativas", en Revista de Derecho Social (Albacete), 80 (2017) 221.

${ }^{41}$ En este sentido, GARRIDO PÉREZ, E., "La representación...”, o. c., p. 224.

${ }^{42}$ Vid., CUADROS GARRIDO, M.E., Trabajadores tecnológicos y empresas digitales, Cizur Menor, Aranzadi, Pamplona 2018, p. 449.

${ }^{43}$ El contenido de este artículo recoge lo previsto en Acuerdo Marco que dispone "el empresario debe respetar la vida privada del teletrabajador. Si existe un medio de vigilancia,
} 
Asimismo, los trabajadores a distancia "deberán cumplir las instrucciones que haya establecido la empresa en el marco de la legislación sobre protección de datos, previa participación de la representación legal de las personas trabajadoras", así como "las instrucciones sobre seguridad de la información específicamente fijadas por la empresa, previa información a su representación legal, en el ámbito de trabajo a distancia" (art. 201 y 2 RD-Ley). De ahí que los sujetos negociadores jueguen un papel fundamental en la regulación del amplio campo que prevé el artículo $87.3 \mathrm{LO} 3 / 2018^{44}$. Cierto es, que cada vez más se van a prestar servicios vía telemática y el empresario va a tener a su disposición una gran variedad de instrumentos de control y vigilancia, los cuales han de ser acotados y transparentes por la negociación colectiva ${ }^{45}$.

A día de hoy, en materia de control de las comunicaciones electrónicas del trabajador las experiencias son muy pocas, ya que el tiempo transcurrido desde la promulgación de la Ley Orgánica y el Real Decreto-Ley es escaso, pero habrá que estar atentos a cómo se desarrollan aquéllas y optar siempre por las buenas prácticas, diferenciando en todo momento la información relacionada con la prestación de sus servicios de la personal.

Asimismo, el uso de dispositivos tecnológicos puestos a disposición por la empresa o con contenido perteneciente a la misma en lugares distintos al centro de trabajo exige que se tenga en cuenta el aspecto relativo a la ciberseguridad de la organización empresarial. De ahí que el artículo 21 Real Decreto-Ley exija a los trabajadores "cumplir con las condiciones e instrucción de uso y conservación establecidas en la empresa en relación con los equipos o útiles informáticos, dentro de los términos que, en su caso, se establezcan en la negociación colectiva". Es decir, los trabajadores han de ser diligentes a la hora de utilizar los medios electrónicos y digitales que la empresa pone a su disposición, conforme a las instrucciones que le marquen al efecto, sobre todo, pensando en la seguridad de la empresa.

\section{CONCLUSIONES}

No cabe duda que la situación vivida durante el año 2020 como consecuencia de la pandemia derivada del Covid-19 aceleró la elaboración del Real Decreto-

\footnotetext{
deberá ser proporcionado al objetivo e introducirse con arreglo a la Directiva 90/270/CEE relativa a las pantallas de visualización”, ya que el artículo 13 ET no recogía nada en particular.

${ }^{44}$ Conforme a este artículo "los empleadores deberán establecer criterios de utilización en los distintos lugares de los dispositivos digitales respetando en todo caso los estándares mínimos de protección de su intimidad de acuerdo con los usos sociales y los derechos reconocidos constitucional y legalmente" y "en su elaboración deberán participar los representantes de los trabajadores".

${ }^{45}$ En este sentido, véase ÁLVAREZ CUESTA, H., "Lugar único, múltiple y ausencias de lugar a partir de la negociación colectiva”, en Documentación Laboral (Madrid), 118 (2019) 110.
} 
Ley que regula el trabajo a distancia, pero no sólo su regulación, sino también los cambios en la forma en que veníamos realizando el trabajo, ya que pasamos al trabajo digital a distancia de un día para otro. Es más, las nuevas formas de prestación $\mathrm{u}$ organización del trabajo se vieron incrementadas exponencialmente por el impacto de la mencionada pandemia, poniendo de manifiesto sus ventajas y debilidades, pero también la necesidad de que se abordara su regulación desde un marco jurídico que aportara seguridad, certeza y transparencia.

Asimismo, todos nos vimos inmersos ante la expansión de la tecnología y su aplicación a una gran variedad de prestaciones de servicios. De repente todos tuvimos que realizar numerosos cursos y adaptarnos al trabajo online o en remoto, sin los conocimientos ni las herramientas necesarios para desarrollarlo de la mejor manera posible.

Nadie duda de las ventajas que tiene el trabajo a distancia o teletrabajo, pero también hay que tener presentes las desventajas o aspectos más negativos. La vigente regulación ha venido para transformar un trabajo presencial en trabajo a distancia y, viceversa.

La prestación de servicios a distantica exige la voluntad concurrente del empresario y la persona trabajadora. A este respecto sería aconsejable una mayor regulación convencional que dotara de un marco adecuado para su desarrollo, evitando la posible invisibilidad del trabajo a distancia

El trabajo a distancia o teletrabajo puede favorecer el derecho a la conciliación conforme al artículo 34.8 ET. A este respecto debe tenerse en cuenta la Directiva 2019/1158 del Parlamento Europeo y del Consejo de 20 de junio de 2019 relativa a la conciliación de la vida familiar y la vida profesional de los progenitores y los cuidadores. Esta Directiva entró en vigor el 1 de agosto de 2019 debiendo realizar su transposición a la normativa española antes del 2 de agosto de 2022, esta norma comunitaria prevé el teletrabajo o trabajo a distancia como herramienta para cubrir las necesidades de conciliación. En este sentido, reconoce "la posibilidad de los trabajadores de adaptar sus modelos de trabajo acogiéndose a fórmulas de trabajo a distancia, calendarios laborales flexibles o reducción de las horas de trabajo (art. 3), si bien, están supeditadas a períodos de trabajo anterior o a una antigüedad que no podrá ser superior a seis meses (art. 9.4).

Ahora bien, la utilización del teletrabajo o trabajo a distancia ha de utilizarse con cuidado, afín de evitar que se convierta en un refugio para las mujeres con cargas familiares, ya que podría llegar a constituir discriminación y menoscabo de las condiciones de trabajo por razón de sexo. Para evitar estas posibles 
situaciones sería necesario que la corresponsabilidad se convirtiera en algo fundamental, sobre todo, para evitar el surgimiento de un nuevo nicho de trabajo precarizado y feminizado. En suma, se debería fomentar más que la conciliación la corresponsabilidad de los hombres y las mujeres frente a las labores de cuidado.

También hay que tener presente que para proteger el derecho a la conciliación de la vida laboral y familiar, es necesario, a su vez, garantizar el derecho a la desconexión digital. El incipiente derecho laboral a la desconexión en nuestro ordenamiento jurídico interno, va a desempeñar un papel relevante en las relaciones laborales, ya que con él, se va a limitar el uso de las tecnologías y se va a garantizar todo lo relativo al tiempo de trabajo (jornada laboral, horarios, fiestas y permisos, así como vacaciones).

Para el trabajador, la desconexión es necesaria en términos de salud, ya que mejoran los niveles de bienestar, felicidad y satisfacción personal, así como disminuyen los estados de depresión y ansiedad. Un trabajador que descansa es un trabajador más productivo y menos propenso a coger bajas laborales, por ello, los empresarios deberían adoptar medidas para que el trabajador cuando termine su jornada laboral, puede descansar mentalmente y disfrutar de su tiempo de ocio haciendo lo que le guste; máxime cuando ello constituye un mandato constitucional, así en virtud del artículo 40.2 se encomienda a los poderes públicos, como uno de los principios rectores de la política social y económica, velar por la seguridad e higiene en el trabajo y garantizar el descanso necesario, limitando la jornada laboral, garantizando las vacaciones periódicas retribuidas y la promoción de centros adecuados, En este sentido, no hay que olvidar que la Organización Mundial de la Salud define la salud como "un estado de completo bienestar físico, mental y social, y no solamente la ausencia de afecciones o enfermedades" ${ }^{\prime 6}$, por consiguiente, no es sólo física sino también mental.

Por ello, urge afín de erradicar la carga mental, diseñar lo mejor posible el puesto de trabajo, conforme a la obligación preventiva de las empresas (art. 15 LPRL y 16 RD-Ley), intentando diseñar puestos de trabajos que no generen situaciones estresantes, organizando los tiempos de trabajo, dando descansos, pausas, flexibilizando, en suma, la jornada laboral, así se aliviaría la carga mental de los trabajadores.

El empresario tiene que proporcionar las herramientas eficaces para un uso de las nuevas tecnologías y ser respetuoso con la seguridad y salud de

\footnotetext{
${ }^{46}$ La cita procede del Preámbulo de la Constitución de la OMS, que fue adoptada por la Conferencia Sanitaria Internacional, firmada el 22 de julio de 1946 por los representantes de 61 Estados y entró en vigor el 7 de abril de 1948.
} 
los trabajadores, mediante la implementación de herramientas tecnológicas para la interrupción efectiva de la comunicación empresa-trabajador o por las que se lleve a cabo el reenvío de demandas laborales exclusivamente hacia empleados en horario de trabajo, así como ha de revisar las cargas de trabajo intentando su ajuste a la jornada pactada. Asimismo, ha de garantizar la formación digital de sus trabajadores, sobre todo, para hacer un uso razonable y saludable de las TICs.

Asimismo, la negociación colectiva va a jugar un papel importantísimo en este sentido, de ahí que los convenios colectivos hayan de dar un mayor tratamiento a este derecho afín de garantizar la seguridad y salud en el trabajo de los riesgos propios de no desconectar de los medios digitales en el trabajo.

Por ello, el Real Decreto-Ley 28/2020, de 22 de septiembre, de trabajo a distancia ha venido a dar respuesta a la necesidad de una regulación legal del trabajo a distancia y el teletrabajo, intentando dar respuesta a todas las cuestiones que se pueden plantear en materia de prevención de riesgos laborales, abono de gastos, formación necesaria para desarrollar la prestación de servicios, control empresarial, brecha de género, jornada de trabajo. Asimismo, habrá que tener en cuenta la regulación convencional sobre estos aspectos.

Lo sabíamos, pero había que demostrarlo. Hoy está claro que trabajar no es calentar la silla. La covid-19 nos ha puesto en nuestro sitio. La pandemia nos ha brindado la oportunidad de acortar aceleradamente un factor clave de la brecha digital que nos separaba de Europa, el del teletrabajo. Para la empresa, los beneficios potenciales son la desvinculación de la fuerza de trabajo de su lugar de residencia, distinto al de la oficina o la fábrica, la atracción de talento de todo el mundo, el ahorro de costes de espacio, o la reducción del absentismo.

Por su parte, a los trabajadores les facilita la conciliación familiar, la organización flexible de su jornada, el acceso a ser contratados por empresas lejanas y el ahorro de tiempo de desplazamientos.

Junto a estas ventajas, no cabe duda que hay inconvenientes, entre otros, mayores dificultades de coordinación y nuevos costes para las empresas, tendencia a la autoexplotación y los horarios abusivos para los trabajadores, así como la pérdida de ventajas de la presencialidad, como la capacidad de los jóvenes de aprender de los veteranos.

En suma, el éxito de la implantación definitiva y generalizada del trabajo digital deberá maximizar sus virtudes y minimizar sus inconvenientes, todos tienen que ganar, empresas y trabajadores. 


\section{BIBLIOGRAFÍA}

- ÁlVAREZ CUESTA, H., "Del recurso al teletrabajo como medida de emergencia al futuro del trabajo a distancia", en Lan Harremanak (BizKaia), 43 (2020) 175-201: https://doi.org/10.1387/lan-harremanak.21722.

- "Lugar único, múltiple y ausencias de lugar a partir de la negociación colectiva", Documentación Laboral (Madrid), 118 (2019) 97-116.

- ÁLVAREZ DEL CUVILLO, A., "El centro de trabajo como unidad electoral: un concepto jurídico indeterminado en un contexto de cambio organizativo", en Nueva Revista Española de Derecho del Trabajo (Pamplona), 188 (2016) 141-177.

- BAQUERO AGUILAR, J., "De la reciente regulación del teletrabajo en las Administraciones Públicas y de la necesidad de una precisa concreción vía negociación colectiva desde la perspectiva de género", en Revista Internacional y Comparada de Relaciones Laborales y Derecho del Empleo (México), ADAPT University Press, Volumen 8, número 4 (2020) 106-145.

- CUADROS GARRIDO, M.E., Trabajadores tecnológicos y empresas digitales, Cizur Menor, Aranzadi, Pamplona 2018.

- DE CASTRO MEJUTO, L.F., "La formación y promoción profesional en el teletrabajo", en Trabajo a distancia y teletrabajo: estudios sobre el régimen jurídico en el derecho español y comparado, Coords. Alicia Villalba Sánchez y Lourdes Mella, Aranzadi, Pamplona 2016, pp. 87-105.

- FERNÁNDEZ PROL, F., "La "conectividad" del trabajador: análisis desde una óptica preventiva", en Temas Laborales. Revista Andaluza de Trabajo y Bienestar (Sevilla), 156 (2021) 149-165.

- GALA DURÁN, C., "La relación entre el teletrabajo y la conciliación de la vida laboral y familiar: el papel de la normativa y la negociación colectiva, en Anuario IET de Trabajo y Relaciones Laborales (Barcelona), 7 (2021) 171185: htpps://doi.org/10.5565/rev/aiet.99.

- GARCÍA JIMÉNEZ, M., "Revolución industrial 4.0, sociedad cognitiva y relaciones laborales: retos para la negociación colectiva en clave de bienestar de los trabajadores", en Revista de Trabajo y de la Seguridad Social (Madrid), CEF, 432 (2019) 147-182. 
- GARRIDO PÉREZ, E., "La representación de los trabajadores al servicio de las plataformas colaborativas", en Revista de Derechos Sociales (Albacete), 80 (2017) 209-232.

- IGARTÚA MIRÓ, T., "El derecho a la desconexión en la Ley orgánica 3/1988, de 5 de diciembre, de protección de datos personales y garantía de los derechos digitales", en Revista de Trabajo y de la Seguridad Social (Madrid) CEF, 432 (2019) 61-87.

- MELLA MÉNDEZ, L., "La seguridad y salud en el trabajo", en Trabajo a distancia y teletrabajo: estudios sobre su régimen jurídico en el Derecho español y comparado, Cizur Menor, Aranzadi, Pamplona 2015, pp. 171-208.

- PASTOR MARTÍNEZ, A., "Trabajo a distancia y derechos colectivos en el Real Decreto-Ley 28/2020 de trabajo a distancia", en Anuario IET de Trabajo y relaciones laborales (Barcelona), 7 (2021) 101-12: htpps://doi.org/10.5565/ rev/aiet.96

- "Una aproximación a la problemática de la representación colectiva de los trabajadores de las plataformas "colaborativas" y en entornos virtuales", en IUS Labor (Barcelona), 2 (2018) 214-233, https://doi.org/10.31009 IUSlabor.2018.io2.06 [consulta 25-5-2021]

- ROJO TORRECILLA, E., "Cambios en el mundo del trabajo. El ejercicio de los derechos colectivos en el entorno empresarial digital y la adaptación sindical", en El nuevo y cambiante mundo del trabajo. Una mirada abierta y crítica a las nuevas realidades laborales": htpp://www.eduardorojotorrecilla.es/ 2021/06/cambios-en-el-mundo-del.trabajo-el.html. 3 de junio de 2021.

- SELMA PENALVA, A., "El accidente de trabajo en el teletrabajo. Situación actual y nuevas perspectivas", en Temas Laborales; Revista andaluza de trabajo y bienestar social_(Sevilla), 134 (2016) 129-166.

- SERRANO GARCÍA, J. M., "Tratamiento del teletrabajo en el Acuerdo Marco Europeo de 16 de julio de 2002", en Relaciones Laborales (Madrid), 2 (2002) 441-462.

- SIERRA BENÍTEZ, E.M., "la retribución, suspensión y extinción en el teletrabajo", en Trabajo a distancia y teletrabajo, Cizur Menor, Aranzadi, Pamplona 2016, pp. 209-240. 
- SUÁREZ QUIÑONES, J.C., "La organización del tiempo de trabajo y descanso y la conciliación en el teletrabajo", en Trabajo a distancia y teletrabajo, Aranzadi, Pamplona 2016, pp. 129-170.

- TASCÓN LÓPEZ, R., "El derecho de desconexión del trabajador (potencialidades en el ordenamiento español)", en Trabajo y Derecho (Madrid), 41 (2018) 45-63.

- "El teletrabajo como forma de presente y futuro de prestación laboral de servicios. Experiencias en la negociación colectiva", en El futuro del Trabajo: cien años de la OIT:comunicaciones, Ministerio de Trabajo, Migraciones y Seguridad Social, Subdirección General de información Administrativa y Publicaciones, Madrid 2019, pp. 1615-1636.

- TRUJILLO PONS, F., "La "desconexión digital" a lomos de la seguridad y salud en el trabajo", en Lan Herremanak (Bizkaia), 45 (2021) 1-18: https://doi.org/10.1387/lan-harremanak.21955. 
